\title{
Management of chronic obstructive pulmonary disease (COPD) in primary care: a questionnaire survey in western Sweden
}

\section{*Jörgen Thornn ${ }^{a, c}$, Maria Norrhall ${ }^{a, d}$, Ronny Larssonc, Dan Curiacc, Gösta Axelsson ${ }^{b}$, Carina Ammonc, Jörgen Månssonn, a, Jonas Brismann , Anna-Lena Söderströmc, Cecilia Björkelund ${ }^{\mathrm{a}, \mathrm{c}}$}

\footnotetext{
a Department of Public Health and Community M edicine/Primary Health Care, The Sahlgrenska Academy at Göteborg University, Sweden

${ }^{b}$ Department of Occupational and Environmental M edicine, The Sahlgrenska Academy at Göteborg University

c Primary Health Care, Västra Götaland Region, Sweden

d Primary Health Care, Halland Region, Sweden
}

Received 3rd April 2007; accepted 12th December 2007

\begin{abstract}
Aim: To assess the primary care management of chronic obstructive pulmonary disease (COPD) in relation to COPD guidelines.

Method: A postal questionnaire was sent out to all Primary Health Care Centres (PHCCs) in western Sweden ( $n=232)$. The response rate was $75 \%$.

Results: A majority of the PHCCs had a nurse and physician responsible for COPD care. They used spirometry equipment regularly, but only $50 \%$ reported that they calibrated it at least weekly. Less than $30 \%$ of the PHCCs reported access to a dietician, occupational therapist or physiotherapist. There was a structured smoking cessation program in $50 \%$ of the PHCCs. Larger PHCCs were more likely to use spirometry equipment regularly and to have specific personnel for COPD care.

Conclusion: There is a need to establish structured programs for COPD care including smoking cessation programs for COPD patients with special trained staff. Larger PHCCs have a better infrastructure for providing guideline-defined COPD care.

(c) 2008 General Practice Airways Group. All rights reserved.

J Thorn, et al. Prim Care Resp J 2008; 17(1): 26-31.

doi:10.3132/pcrj.2008.00008
\end{abstract}

Keyw ords practice nurses, COPD, primary health care, spirometry, multidisciplinary approach

\section{Introduction}

Chronic Obstructive Pulmonary Disease (COPD) is an increasing health problem. In the year 2020, COPD may be the third most common cause of death worldwide. ${ }^{1}$ The presence of obstructive lung disease has been shown to be a significant predictor of earlier death in long-term follow up. ${ }^{2}$ About $8 \%$ of the population aged 46 years and over in Sweden have COPD. ${ }^{3}$ The major causative agent is smoking, but different occupational exposures can also cause COPD. 4,5

In Sweden, Primary Health Care Centres (PHCCS) manage the majority of COPD patients. In recent years, special "asthma nurse practices" have been established in PHCCs, but it is unclear to what extent COPD patients attend these special practices. There are reported improvements in COPD patient care in nurse-led primary health care clinics in the UK. ${ }^{6,7}$

Several previous studies have shown that COPD is underdiagnosed..$^{8,9}$ There are reports suggesting that general practitioners (GPs) seldom use spirometry for diagnosing COPD among smokers with respiratory symptoms. ${ }^{10}$ However, it has been shown that it is possible to identify COPD patients early by active spirometry screening in primary care and there are rapidly growing resources for this in Sweden. ${ }^{9,11}$ In addition, there are a few recently published studies from different

\footnotetext{
* Corresponding author: Department of Public Health and Community Medicine/Primary Health Care, The Sahlgrenska Academy at Göteborg University, Box 454, 40530 Gothenburg, Sweden. Tel: +46 317736828 Fax: +46 317781704 E-mail: jorgen.thorn@allmed.gu.se
} 
countries indicating gaps in primary health care physicians' knowledge of COPD management. ${ }^{12-14}$

The size of the PHCC - which is the basic unit of the primary health care system in Sweden - may be an important factor in the provision of COPD care, and this variable has previously been considered in data analysis among patients with asthma. ${ }^{15}$ Each PHCC is either responsible for a defined population, or for patients who register there, and the respective patient group is used to define the size of the PHCC. In a previous study, using this definition, larger PHCCs were more likely to have an "asthma nurse practice" as compared to smaller PHCCs. ${ }^{16}$

Consequently, we hypothesised that there should be considerable potential for improvement in the primary care management of COPD in Sweden. National COPD guidelines have recently been published; ${ }^{17}$ these are based on, and are similar to, other international COPD guidelines such as the Global Initiative for Chronic Obstructive Lung Disease (GOLD) guideline. ${ }^{18}$

Therefore, the purpose of the present study was to assess COPD management in the primary health care system in western Sweden in relation to recently-published national guidelines, and to assess COPD care in relation to PHCC size. It was hoped that the results of this study could be used to compare standards of COPD management between different countries and cultures, and to improve guideline-defined COPD management in primary care.

\section{Methods}

All PHCCs in western Sweden $(n=232)$ were contacted and invited to participate in the postal questionnaire survey of COPD management. Four reminders (three postal reminders and a final reminder by phone) were provided. The questionnaires were addressed to the principal manager of the centre. If the centre had a GP or nurse responsible for COPD care, the principal manager of the centre was assisted by them in answering the questionnaire. The PHCCs included in the study were either responsible for a defined population within a certain geographic area (population-based: the traditional PHCC in Sweden) or had patients registered with the respective GP (registration-based).

\section{Questionnaire}

The postal questionnaire was based on previous similar questionnaires used in different regions in Sweden ${ }^{16}$ aimed at studying COPD care within the primary health care system. The questions were focused on COPD care as per COPD guidelines. ${ }^{17,18}$ The final questionnaire contained a series of items on the healthcare professionals at the PHCC, the population characteristics of the patients covered by the PHCC, and whether the PHCC was population-based or registation-based. These were followed by a series of questions on the presence of nurse-led asthma or COPD practices and the use of spirometry. The questionnaire concluded with knowledge questions on the national COPD guidelines.

\section{Statistical analyses}

Descriptive statistics were used to process prevalence data. The differences between PHCCs with different population characteristics and sizes were analysed using non-parametric tests (chi ${ }^{2}$ Fisher's exact test). In the analysis comparing different PHCCs with respect to size, the cut-off level (more or less than 8000 inhabitants) was determined according to the number of inhabitants in each statistical group - i.e. we aimed to compare groups which were as equal in "numbers (n)" as possible. Differences were considered statistically significant at $p<0.05$.

The Ethics Committee of the Faculty of Medicine at Göteborg University approved the study.

\section{Results}

The response rates for the different PHCCs are shown in Table 1. There was a difference in response rate between population-based and registration-based PHCCs. The highest response rate was found among population-based PHCCS. Two PHCCs were excluded from the analysis since incomplete answers were given.

Table 2 shows the background characteristics of the PHCCs. A majority of the PHCCs had a nurse and a physician responsible for COPD care. Most PHCCs reported that they had spirometry equipment which was used regularly. Approximately $90 \%$ of the PHCCs reported that they used the spirometry equipment at least several times a week, but only $50 \%$ reported that they calibrated it at least once a week (in $19.5 \%$ of the PHCCs, the spirometry equipment was calibrated every other day). Less than $15 \%$ reported access to a dietician and $25-35 \%$ had access to a physiotherapist and/or an occupational therapist for COPD patients.

Table 3 shows the data on COPD management at the PHCCs. About $80 \%$ of the PHCCs reported that they usually used spirometry to investigate smokers with current respiratory symptoms. About half of the PHCCs had a

Table 1. Questionnaire response rates.

\begin{tabular}{lccc} 
& $\begin{array}{c}\text { Responders } \\
\mathrm{n}\end{array}$ & $\begin{array}{c}\text { Sent by mail } \\
\mathrm{n}\end{array}$ & $\begin{array}{c}\text { Response rates } \\
\%\end{array}$ \\
\hline $\begin{array}{l}\text { All PHCCs } \\
\begin{array}{l}\text { Population-based } \\
\text { PHCCs }\end{array}\end{array}$ & 173 & 232 & 75 \\
$\begin{array}{l}\text { Registration-based } \\
\text { PHCCS }\end{array}$ & 150 & 183 & 82 \\
\end{tabular}


Table 2. Background characteristics of the Primary Health Care Centres (PHCC). Results are presented for the total number of PHCCs, and for population-based and registration-based PHCCS.

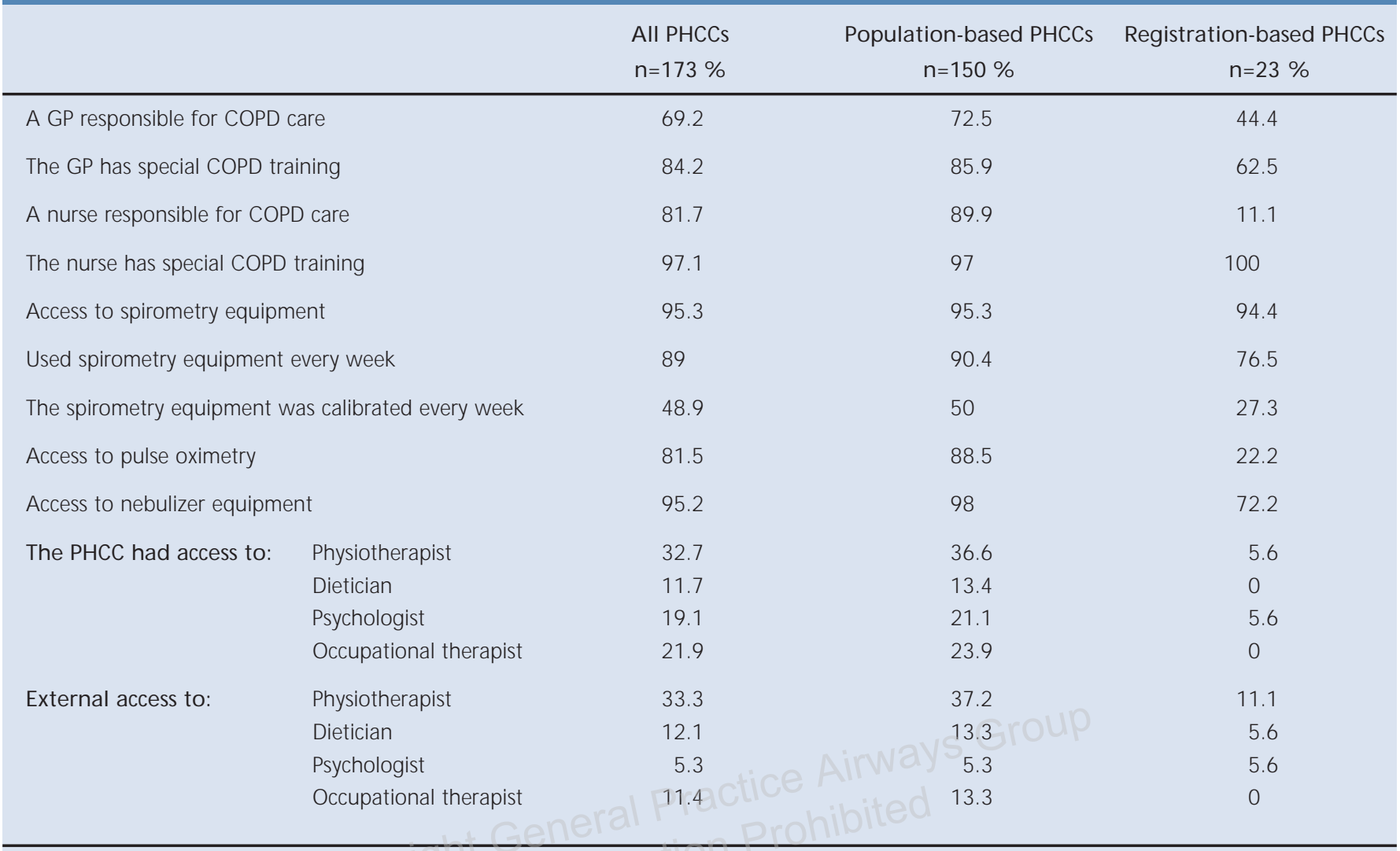

COPD = Chronic Obstructive Pulmonary Disease; PHCC = Primary Health Care Centre; GP = General Practitioner

Table 3. Chronic Obstructive Pulmonary Disease (COPD) management at the Primary Health Care Centres (PHCC). Results are presented for the total number of PHCCs, and for population-based and registration-based PHCCs.

$\begin{array}{ccc}\text { All PHCCS } & \text { Population-based PHCCS } & \text { Registration-based PHCCS } \\ n=173 \% & n=150 \% & n=23 \%\end{array}$

Used spirometry on smokers with current respiratory

symptoms

Smoking cessation program:

The PHCC had an organised and structured smoking

cessation program involving special trained staff

No smoking cessation program due to lack of resources

51.2

COPD care:

Had an organised and structured program in special

COPD nurse practices

Measurements of bone mineral density

A structured vaccination program offered to the

COPD patients

Knowledge about the national COPD guidelines
14.2

COPD = Chronic Obstructive Pulmonary Disease; PHCC = Primary Health Care Centre; GP = General Practitioner 
Table 4. Chronic obstructive pulmonary disease (COPD) management in relation to PHCC size, based on the number of inhabitants in the PHCC's geographic area (more or less than 8000 inhabitants).

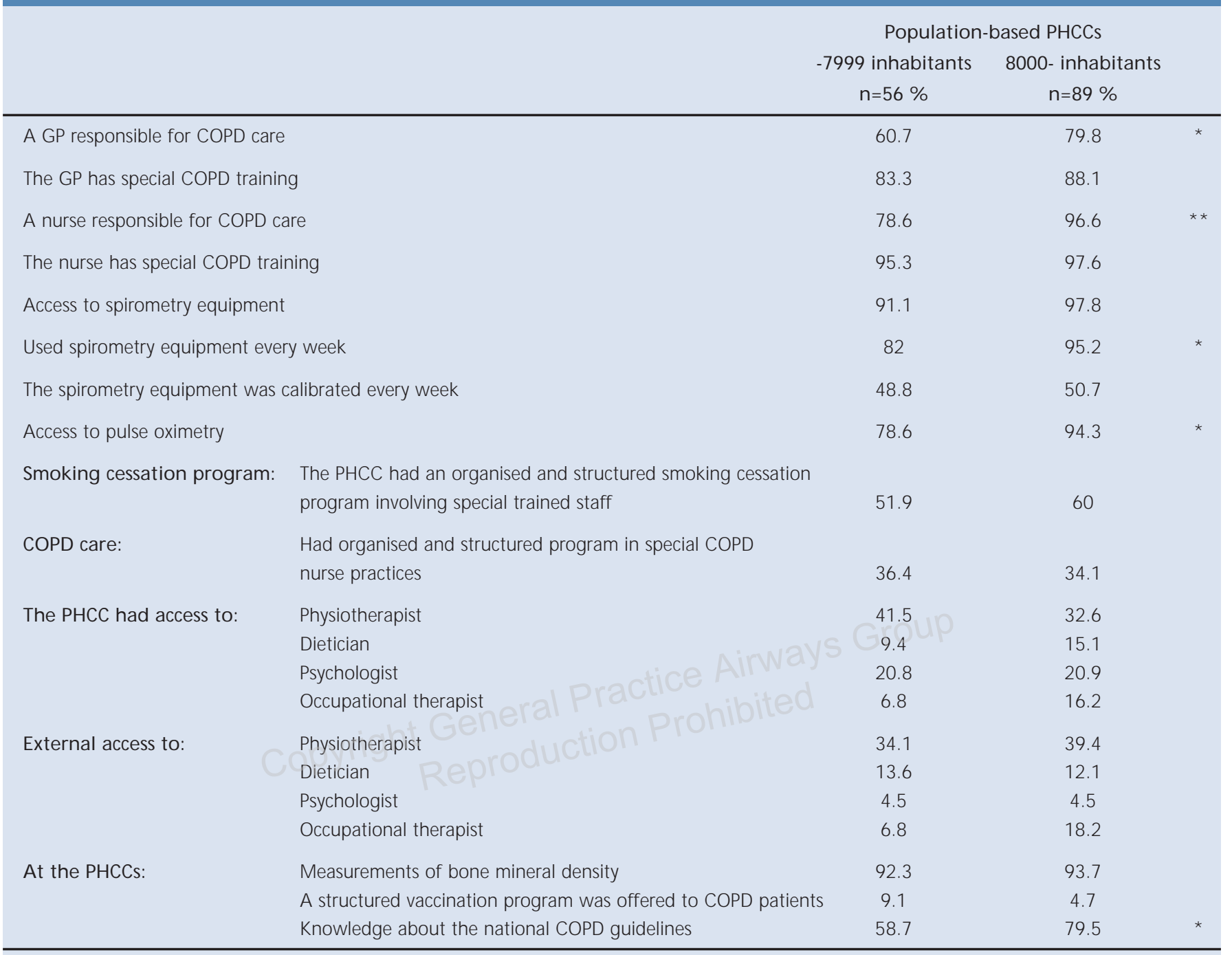

$* p<0.05 ; * *<<0.01 ; C O P D=$ Chronic Obstructive Pulmonary Disease; PHCC = Primary Health Care Centre; GP = General Practitioner

structured smoking cessation program. A structured program for COPD management was established in a minority of the PHCCs. In a minority of the PHCCs, vaccination programs and measurements of bone mineral density were used on a regular basis in structured programs for COPD patients. Most of the PHCCs reported knowledge about the national COPD guidelines.

The results for the registration-based PHCCs differed in some respects. However, the number of PHCCs in this group was small and the results must therefore be interpreted with care.

Table 4 shows the data on COPD management in relation to PHCC size for population-based PHCCs (the traditional PHCC in Sweden). A statistically significant higher proportion of the larger PHCCs had a nurse and a physician responsible for COPD care and a higher proportion reported knowledge about the national guidelines for COPD, compared to the smaller PHCCs. A higher proportion of the larger PHCCs had access to pulse oximetry and used their spirometry equipment regularly.

\section{Discussion}

\section{Difficulties encountered during the study}

The results were based on self-reporting of COPD management at PHCCs. Staff at the PHCCs with organised/structured COPD care may have been more prone to answer the questionnaire, and responders may have been more willing to express a positive view of COPD care. Thus, there is a possibility that over-reporting and positive selection are sources of bias in this type of questionnaire survey. 
However, in this study the overall response rate was high $(75 \%)$, indicating that the results were representative of COPD management in primary health care. Furthermore, the response rate was even higher (82\%) among the populationbased PHCCs (which were analysed separately, as they represent the traditional PHCC in Sweden). Selection bias should, therefore, not be a problem in the analyses representing the main results of the study.

Regarding registration-based centres, separate analyses were performed since the response rate was lower and the number of centres smaller, as compared with the other population-based PHCCs. However, previous studies indicate the possibility of no bias even with response rates as low as $30 \% .{ }^{19}$ These results must be interpreted with care.

\section{New questions arising from the study}

Spirometry is the "gold standard" for COPD diagnosis. In this study, almost all of the PHCCs reported that they had spirometry equipment which was used regularly (>90\% used it at least several times a week). In a previous study from midSweden, $77 \%$ of the PHCCs had access to a spirometer. ${ }^{16}$ However, in this study, only half of the PHCCs calibrated their spirometry equipment at least once a week. If the equipment is not regularly calibrated, the spirometry readings may be imprecise, thus affecting the COPD diagnostic procedure. The results of this study suggest a need for information and training in this respect.

The major causative agent of COPD is smoking and smoking cessation programs can thus be powerful tools in the treatment and prevention of the disease. In this study, about half of the PHCCs had established a structured smoking cessation program with specially trained staff. This is encouraging, since previous studies in Belgium and Switzerland have reported that a minority of the PHCCs used structured programs for COPD patients. ${ }^{12,13}$ However, the effectiveness of the smoking cessation programs is not clear. There is a need to evaluate these programs in future studies.

A multidisciplinary approach is recommended in several international COPD guidelines in order to establish organised programs for COPD care, but less than $30 \%$ of the PHCCs in the study reported access to a dietician, occupational therapist or physiotherapist. There are previous studies showing improvement in COPD patient care in special structured nurse-led clinics within a primary health care system $^{6,7}$ and structured physical training programs may have a positive effect on dyspnoea and quality of life in COPD patients. ${ }^{20,21}$ The results of this study showed that there was some access to dieticians, occupational therapists and physiotherapists for COPD patients in the primary health care system, but there is still room for improvements in the multidisciplinary approach with respect to establishing guideline-defined structured programs for COPD care.
A higher proportion of the larger PHCCs had a nurse and a physician responsible for COPD care, and a higher proportion reported knowledge about the national COPD guidelines, compared to the smaller PHCCs. These results are in accordance with previously published data from PHCCs in mid-Sweden. ${ }^{16}$ The results also indicate that larger PHCCs may be more prone to use spirometry regularly; this is noteworthy, since spirometry is vital for the diagnosis of COPD. These data show that larger PHCCs may have a better COPD infrastructure, and that a certain size of PHCC may facilitate guideline-defined COPD care.

\section{Conclusions}

This study suggests that there is still room for more information and training regarding spirometry, which is vital for diagnosing COPD. The spirometry equipment must be calibrated regularly. There is a need to establish structured programs for COPD care including smoking cessation programs for COPD patients with special trained staff. The effectiveness of this approach must be evaluated regularly according to COPD guidelines. Furthermore, larger PHCCs have a better infrastructure for providing COPD care in accordance with national and international guidelines.

\section{Funding acknow ledgements}

This study was supported by funds from the Research Councils of Region Västra Götaland and Halland, the Swedish Association of General Practice, and the Göteborg Medical Society.

\section{Conflict of interest declaration}

None declared.

This data has previously been presented at the following meetings:

Swedish Society of Medicine Annual Meeting, Stockholm, Sweden, December 2005

International Primary Care Respiratory Group (IPCRG) Meeting, Oslo, Norway, June 2006.

\section{References}

1. Gulsvik A. The global burden and impact of chronic obstructive pulmonary disease worldwide. Monaldi Arch Chest Dis 2001;56(3):261-4.

2. Mannino DM, Buist AS, Petty $T L$, Enright PL, Redd SC. Lung function and mortality in the United States: data from the First National Health and Nutrition Examination Survey follow up study. Thorax 2003;58(5):388-93.

3. Lundback $B$, Lindberg $A$, Lindstrom $M$, et al. Not 15 but $50 \%$ of smokers develop COPD?--Report from the Obstructive Lung Disease in Northern Sweden Studies. Respir Med 2003;97(2):115-22.

4. Balmes J, Becklake M, Blanc P, Henneberger P, et al. American Thoracic Society Statement: Occupational contribution to the burden of airway disease. Am J Respir Crit Care Med 2003;167(5):787-97.

5. Becklake MR. Occupational exposures: evidence for a causal association with chronic obstructive pulmonary disease. Am Rev Respir Dis 1989;140(3 Pt 2):S85-91.

6. Shirley K, Kelly R. COPD management within primary care. Nurs Times 2002;98(26):52-3.

7. Stothard A, Brewer K. Dramatic improvement in COPD patient care in nurseled clinic. Nurs Times 2001;97(24):36-7. 
copyright GPAG - Reproduction prohibited

8. Petty TL. Scope of the COPD problem in North America: early studies of prevalence and NHANES III data: basis for early identification and intervention. Chest 2000;117(5 Suppl 2):326S-31S

9. Nihlen $U$, Montnemery $P$, Lindholm LH, Lofdahl CG. Detection of chronic obstructive pulmonary disease (COPD) in primary health care: role of spirometry and respiratory symptoms. Scand J Prim Health Care 1999;17(4):232-7.

10. den Otter JJ, van Dijk B, van Schayck CP, Molema J, van Weel C. How to avoid underdiagnosed asthma/chronic obstructive pulmonary disease? J Asthma 1998;35(4):381-7.

11. Lundqvist G, Persson A, Widman L, Lundgren R, Linden G. [Chronic obstructive lung disease. Early diagnosis in primary health care requires well-functioning spirometry routines]. Lakartidningen 2001;98(43):4702-03.

12. Decramer M, Bartsch P, Pauwels R, Yernault JC. Management of COPD according to guidelines. A national survey among Belgian physicians. Monaldi Arch Chest Dis 2003;59(1):62-80.

13. Rutschmann OT, Janssens JP, Vermeulen B, Sarasin FP. Knowledge of guidelines for the management of COPD: a survey of primary care physicians. Respir Med 2004;98(10):932-7.

14. Minas M, Dimitropoulos K, Pastaka C, Papadopoulos D, Markoulis N, Gourgoulianis Kl. Global initiative for chronic obstructive lung disease for chronic obstructive pulmonary disease: GOLD opportunity for lung disorders. Prev Med 2005;40(3):274-7.

15. Hasselgren M, Gustafsson D, Ställberg B, Lisspers K, Johansson G. Management, asthma control and quality of life in Swedish adolescents with asthma. Acta Pediatrica 2005;94:1-7.

16. Lisspers K, Ställberg B, Hasselgren M, Johansson G, Svärdsudd K. Organisation of asthma care in primary health care in mid-Sweden. Prim Care Resp J 2005; 14(3):147-53. doi:10.1016/j.pcrj.2005.03.003

17. SLMF. The Swedish Society of Respiratory Medicine, National guidelines (in Swedish). In. www.slmf.se; 2003/2005.

18. GOLD. Global initiative for chronic Obstructive Lung Disease (GOLD); Global strategy for the diagnosis, management, and prevention of chronic obstructive pulmonaru disease; NHLBI/WHO Workshop. In; 1998/2005. Available at www.goldcopd.com

19. Nohr EA, Frydenberg M, Henriksen TB, Olsen J. Does low participation in cohort studies induce bias? Epidemiology 2006;17(4):413-18.

20. Emery CF, Schein RL, Hauck ER, Maclntyre NR. Psychological and cognitive outcomes of a randomized trial of exercise among patients with chronic obstructive pulmonary disease. Health Psychol 1998;17(3):232-40.

21. Jones RC, Copper S, Riley O, Dobbs F. A pilot study of pulmonary rehabilitation in primary care. Br J Gen Pract 2002;52(480):567-8.

\section{Available online at http://w w w.thepcrj.org}

